\title{
The President's Page
}

\section{Opening Frontiers at Home}

\author{
Antonio Carlos Palandri Chagas \\ President of SBC
}

As we open frontiers for the national cardiology, I am very happy to announce to the members of the Brazilian Society of Cardiology (SBC), the discovery of an unexplored territory: the African continent.

At the end of January, by invitation of the President of the Portuguese Society of Cardiology, Hugo Madeira, we went to the city of Praia, capital of Cabo Verde, to attend the 1st Jornadas Lusófonas de Cardiologia. The Brazilian team was in charge of five presentations, which dealt on valvular cardiopathy and coronary artery disease.

In the same month, representatives from the Study Group on Pulmonary Circulation of SBC went to Mexico to participate in the third annual meeting of the Pulmonary Vascular Research Institute, with which SBC has already established an exchange of knowledge. Moreover, for the first months of this year, we have already confirmed our participation in three other international forums: the 2009 ACC Annual Scientific Session, the XXX Portuguese Congress of Cardiology and the XXII Peruvian Congress of Cardiology.

In Orlando, the city where the event sponsored by the American College of Cardiology will be held, we will have a joint symposium with the entity for the second time. The session, to be held March 30 at 12:15 PM, demonstrates the success of its former version, held in Chicago last year.

In addition to North-American specialists, the schedule will include three conferences given by Brazilian professionals: "The Impact of cardiovascular disease in Brazil and SBC Initiatives in Risk Control"; "Optimal Treatment of Acute Coronary Syndromes" and "Revascularization: Lessons from the SYNTAX Study".

After Cabo Verde and the USA, at the end of April, SBC will be in Portugal and in Peru, representing National Cardiology in the congresses sponsored by the Societies of these two countries.

At the national level, the SBC Executive and Scientific Congress Committee is working at full speed, preparing for the 64th Brazilian Congress of Cardiology, which will be held September 12-16 in Salvador, Bahia. News coming soon!

Within the same perspective, of offering our members the opportunity to undertake highquality refresh courses, the inclusion of the "Directives under Debate" project is predicted for the congress schedule of 10 State Societies. That is a fast and efficient scientific activity for the qualification of the content of the documents selected for discussion. The coordination in charge of this division works not only to promote the directives. The current Board is at present preparing 28 new documents for publication. And, in order to include these more recent directives to the protocols of the Ministry of Health associated to the area of cardiology, SBC is working full-time with the government. The restructuring will benefit the broad universe of patients that use the public health services.

The prominent presence of the entity, working together with the Federal Government, has been a growing focus among the priorities of this Board, either by assisting the projects and decisions of cardiovascular policy, or by obtaining funding sources for research and, mainly, by preparing and defending better working conditions for the Brazilian cardiologist. In doing so, $\mathrm{SBC}$ is fulfilling its social and associative mission!

Mailing address: Antonio Carlos Palandri Chagas •

Avenida Marechal Câmara, 160/330, Centro - 20020-907, Rio de Janeiro, RJ - Brazil

E-mail: acchagas@cardiol.br 